\title{
Solid State Nuclear Magnetic Resonance as a Tool to Explore Solvent-Free MALDI Samples
}

\author{
Hélène Pizzala, ${ }^{a}$ Caroline Barrère, ${ }^{a}$ Michaël Mazarin,, ${ }^{a}$ Fabio Ziarelli, \\ and Laurence Charles ${ }^{a}$ \\ ${ }^{a}$ Universités Aix-Marseille I, II \& III - CNRS, UMR 6264: Laboratoire Chimie Provence, Spectrométries \\ Appliquées à la Chimie Structurale, Marseille, France \\ b Fédération des Sciences Chimiques - CNRS, FR1739, Spectropole, Marseille, France
}

Solid-state Nuclear magnetic resonance (NMR) was used here to explore structural characteristics of samples to be subjected to matrix-assisted laser desorption/ionization (MALDI) and prepared without the use of any solvent. The analytical systems scrutinized in NMR were mixtures of a 2,5-dihydroxybenzoic acid (2,5-DHB) matrix and caesium fluoride $(\mathrm{CsF})$, used as the cationization agent in synthetic polymer MALDI mass analysis, at different molar ratios (1:1, 5:1, and 10:1). Complementary information could be obtained from ${ }^{13} \mathrm{C}$, ${ }^{133} \mathrm{Cs}$, and ${ }^{19} \mathrm{~F}$ NMR spectra. Grinding the matrix together with the salt in the solid state was shown to induce a strong modification in the molecular organization within the MALDI sample. The evidenced mechano-induced reactions allow strong interactions between the matrix and the cation, up to the formation of a salt, and only occur in the presence of some water molecules. Addition of a poly(ethylene oxide) polymer as the analyte did not further modify the observed molecular organizations. Although relative matrix and salt concentrations in the scrutinized samples were unusual for MALDI analysis, mass spectra of good quality could be obtained and revealed that cation attachment on polymers during the MALDI process is not a matrix-independent event since a lower ionization efficiency was obtained from highly organized solid samples, mostly consisting of 2,5-DHB caesium salt species. (J Am Soc Mass Spectrom 2009, 20, 1906-1911) (C) 2009 American Society for Mass Spectrometry

$\mathrm{M}$ atrix-assisted laser desorption/ionization (MALDI) is widely used as an efficient ionization method to characterize synthetic polymers by time-of-flight mass spectrometry (TOF MS) [1, 2]. A key point in the success of a MALDI-MS analysis is sample preparation, that is, proper matrix and cation selection as well as solid-state organization. The matrix actually plays multiple roles in the MALDI process and should accordingly present specific properties. Some criteria are clearly defined, such as a strong absorptivity at the employed laser wavelength or good vacuum stability, while other requirements, such as a good miscibility of the matrix with the analyte in the solid state, are not easily related to physico-chemical parameters of the matrix. As a result, prediction for the optimal matrix-polymer system is still not possible and sample preparation methods are usually developed from published protocols that were shown to work for a given polymeric system [3]. In particular, solvent-free MALDI was recently proposed as an alternative to the conventional dried droplet method [4,5].

Despite MALDI being a widely used technique, mechanisms underlying this desorption/ionization process are

Address reprint requests to Dr. L. Charles, Spectrométries Appliquées à la Chimie Structurale, Campus Saint-Jérôme, Case 511, 13397 Marseille Cedex 20, France. E-mail: laurence.charles@univ-provence.fr and helene.pizzala@ univ-provence.fr still not fully understood. To investigate the MALDI process, many studies have focused on sample morphology using different imaging experiments. Sample heterogeneity and distribution of molecules within the MALDI solid sample have been explored using microscopy techniques, such as optical microscopy [6, 7], scanning electron microscopy [8-12], atomic force microscopy [12], and confocal laser scanning microscopy $[13,14]$. Spectroscopic techniques, such as fluorescence spectrophotometry [13] or Raman spectrometry [8] were also implemented in conjunction with microscopy to obtain complementary information. Imaging techniques based on mass spectrometry $[12,15,16]$ were also used to evidence segregation phenomenon within the sample. X-ray diffraction was performed to reach atomic level information [17, 18]. A similar approach, involving various imaging techniques, has recently been reported to investigate the morphology of solventfree MALDI samples [19].

Solid-state nuclear magnetic resonance (NMR) has proven to be a valuable spectroscopic method to characterize solid-state structure at the atomic level. Currently, using state of the art techniques, such as magic angle spinning (MAS) [20] to increase resolution, and cross polarization (CP) [21] to improve sensitivity, it is possible to obtain high-resolution solid-state NMR spectra similar to those obtained in the liquid state. 
Chemical shift is extremely sensitive to local changes in the structure, and differences in the NMR spectra can be detected for samples that are identical for other solidstate techniques. NMR spectra are influenced by the local nucleus environment, extending to few spheres of bonding, so long range order is not required. Although amorphous materials spectra tend to display broader lines than polycrystalline powder, NMR is able to explore various morphological systems. In particular, the use of solid-state NMR was very recently reported to test the influence of crystallization conditions on MALDI sample morphology [22]. Moreover, a main advantage of this technique is its ability to access many different nuclides, so that data from different elements in a given sample provide complementary information.

In this work, the ability of solid-state NMR to explore structural characteristics of MALDI samples has been evaluated. So far, most studies have addressed the interaction between the matrix and the analyte, and little information has been reported on the possible interaction between the matrix and the cationization agent, which is required in the case of synthetic polymer ionization. Although cation attachment in MALDI of polymers was reported to be largely a matrix-independent event [23], the presence of specific functional groups (such as $\mathrm{OH}$ or $\mathrm{COOH}$ ) was shown to be very important for the cationization process [24]. The present study is focused on solvent-free MALDI samples consisting of 2,5-dihydroxybenzoic acid (2,5-DHB) and caesium fluoride ground together in the solid state at different molar ratios. This particular matrix/salt system was selected as it is currently used in our laboratory for MALDI of large poly(ethylene oxide) polymers, and because it allows complementary NMR data to be obtained from ${ }^{13} \mathrm{C},{ }^{133} \mathrm{Cs}$, and ${ }^{19} \mathrm{~F}$ spectra.

\section{Experimental}

\section{Chemicals}

The matrix, 2,5-dihydroxybenzoic acid (2,5-DHB), and the cationization agent, caesium fluoride $(\mathrm{CsF})$, were obtained from Sigma-Aldrich (St. Louis, MO, USA). The tested polymer sample was poly(ethylene oxide) (PEO) with $M_{n} 1790$ from Sigma Aldrich. External mass calibration was performed with polystyrene $M_{n} 1790$ from Sigma-Aldrich.

\section{Sample Preparation}

Matrix and salt mixtures analyzed in solid-state NMR were prepared by grinding 2,5-DHB and $\mathrm{CsF}$ in a mortar during $10 \mathrm{~min}$ in different matrix/salt molar ratios (1:1, 5:1, and 10:1). To study the influence of the analyte on the matrix/salt solid mixture by NMR, as well as to perform MALDI analysis, the PEO polymer was added to the matrix/salt solid mixture to reach a matrix/polymer molar ratio of about 250:1. For preparation in a dry nitrogen atmosphere, the mixtures were ground in a glove box after heating 2,5-DHB, CsF, and polymer under vacuum to remove residual water. Note that NMR data obtained for so-dried materials (data not shown) did not evidence any modification due to this thermal treatment. For MALDI analysis, a few grains of the solid mixture were applied to the MALDI target and pressed with a small spatula to form a thin film.

\section{Mass Spectrometry}

MALDI-TOF MS experiments were carried out using a Bruker Autoflex (Bruker Daltonics, Leipzig, Germany). The instrument is equipped with a nitrogen laser emitting at $337 \mathrm{~nm}$, a single-stage pulsed ion extraction source, and dual microchannel plate detectors. Data acquisition was performed in reflectron mode. Positive ion mode was used for all analyses, using a pulse frequency of $10 \mathrm{~Hz}$ and an accelerating voltage of $19 \mathrm{kV}$. The delay time used in delayed extraction mode was optimized based on the mass range of the polymer distribution and was generally around 100 ns. FlexControl software version 2.2 (Bruker Daltonics) was used for instrument control and data acquisition, and FlexAnalysis software version 2.2 (Bruker Daltonics) for data processing.

\section{Solid-State NMR Spectroscopy}

Solid-state NMR spectra were obtained on a Bruker Avance DSX 400 (9.4T) spectrometer. ${ }^{19} \mathrm{~F}$ MAS experiment spectra were recorded using a $2.5 \mathrm{~mm}$ double channel Bruker probehead, spinning at $25 \mathrm{kHz} .{ }^{13} \mathrm{C}$ and

${ }^{133} \mathrm{Cs}$ experiments were acquired on a $4 \mathrm{~mm}$ double channel Bruker probehead, with a spinning rate of 8 to $10 \mathrm{kHz} .{ }^{13} \mathrm{C}$ were observed using variable amplitude cross polarization and magic angle spinning (CPMAS). Detailed experimental set-up is provided as Supplementary Material, which can be found in the electronic version of this article. Chemical shifts were referenced with respect to tetramethylsilane (set to $0 \mathrm{ppm}$ ) for ${ }^{13} \mathrm{C}$, to a $0.1 \mathrm{M} \mathrm{KF}(\mathrm{aq})$ solution (set to $-119 \mathrm{ppm}$ ) for ${ }^{19} \mathrm{~F}$ and to $0.5 \mathrm{M} \mathrm{CsCl}$ (aq) solution (set to $0 \mathrm{ppm}$ ) for ${ }^{133} \mathrm{Cs}$. NMR spectral simulations were made using DMFIT software [25].

\section{Results and Discussion}

\section{Solid-State NMR}

Solid-state NMR experiments were first performed on starting material samples. ${ }^{19} \mathrm{~F}$ and ${ }^{133} \mathrm{Cs}$ MAS spectra of caesium fluoride are shown in Figure $1 \mathrm{a}$, while the ${ }^{13} \mathrm{C}$ CPMAS spectrum of 2,5-dihydroxybenzoic acid is displayed Figure 2a. Caesium fluoride is characterized by a ${ }^{19} \mathrm{~F}$ signal at $-14 \mathrm{ppm}$ and a ${ }^{133} \mathrm{Cs}$ signal at $175 \mathrm{ppm}$, as reported [26, 27]. On both ${ }^{19} \mathrm{~F}$ and ${ }^{133} \mathrm{C}$ s MAS spectra, weak resonances are observed, and have been ascribed to surface hydrated species [27, 28]. These signals disappear as residual water molecules are removed after heating the sample under vacuum. The ${ }^{13} \mathrm{C} C \mathrm{CPAS}$ spectrum 
(a)

${ }^{19} \mathrm{~F}$

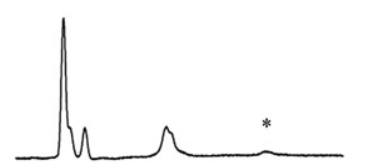

(b)

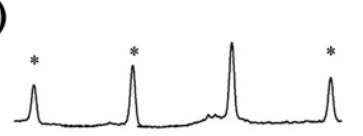

(c)

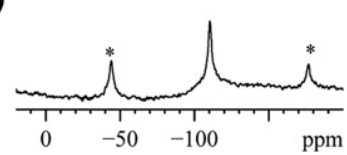

${ }^{133} \mathrm{Cs}$
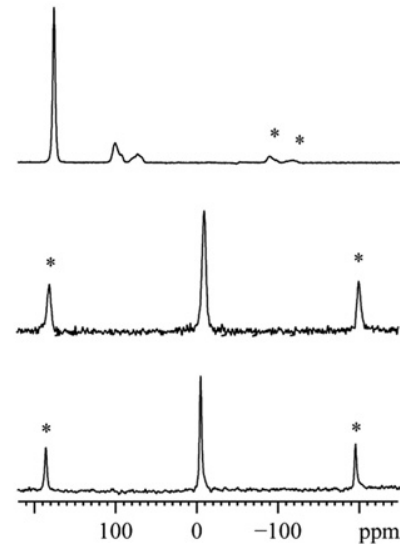

Figure 1. ${ }^{19} \mathrm{~F}$ (left) and ${ }^{133} \mathrm{Cs}$ (right) MAS NMR spectra of (a) CsF, and 2,5-DHB/CsF ground mixtures in (b) 1:1 and (c) 5:1 molar ratio. *Spinning side bands.

of 2,5-dihydroxybenzoic acid displays seven resonances associated to seven carbon atoms, indicating a single type of molecule in the asymmetric unit. In the solidstate, carboxylic acids form centrosymmetric dimers, linked by hydrogen bonds between carboxylic groups (as illustrated in the inset of Figure 2a). In 2,5-DHB, dimers are stacked to form columns, which are held together by hydrogen bonds involving hydroxylic groups in position 5 to form sheets [29]. This particular organization explains the shifts observed in the solidstate experiments (compared with liquid state NMR data), in particular for signals of carboxylic (C7) and both phenol carbons (C2 and $\mathrm{C} 5$ ), which are characteristic of the strong intra- and intermolecular hydrogen bonds in the crystal.

After grinding the matrix and the salt, NMR spectra are strongly modified. For example, while signals of $\mathrm{CsF}$ and hydrated species disappear, new high field shifted resonances are observed in both ${ }^{133} \mathrm{Cs}$ and ${ }^{19} \mathrm{~F}$ spectra. However, observed differences were also shown to depend on the matrix/salt molar ratio in the studied samples. The investigated mixtures were prepared using quite low matrix/salt ratios (i.e., 1:1, 5:1, and 10:1) with regards to usual MALDI experimental conditions, to improve the sensitivity of NMR ${ }^{13} \mathrm{C}$ experiments.

For the 1:1 matrix/salt molar ratio, the single peak resonance observed in the ${ }^{19} \mathrm{~F}$ NMR spectra is strongly shifted down to $-126 \mathrm{ppm}$ (Figure 1b), and would be consistent with a $\mathrm{F}^{-}$species strongly interacting with $\mathrm{H}_{3} \mathrm{O}^{+}[28,30]$. Similarly, the chemical shift measured for the ${ }^{133}$ Cs NMR signal is high field shifted compared with the value obtained for the pure salt sample. The strong shift of both the anion and cation signals gives clear evidence of a dramatic modification in nucleus local environments. In particular, the high field shift in ${ }^{133}$ Cs NMR spectrum is consistent with a coordination of the $\mathrm{Cs}^{+}$cation by oxygen atoms. Chemical shift measured in Figure $1 \mathrm{~b}$ suggests approximately eight to ten oxygen atoms around the $\mathrm{Cs}^{+}$cation [31], which is consistent with the irregular coordination sphere of $\mathrm{Cs}^{+}$ ion reported for caesium salicylate crystal [32], exclusively consisting of eight oxygen atoms. However, although CsF is a highly hygroscopic salt, observed ${ }^{133} \mathrm{Cs}$ chemical shifts are neither compatible with surface hydrated species (expected at $74 \mathrm{ppm}$ and 101 ppm) nor with fully hydrated species (usually observed near $45 \mathrm{ppm}$ ). The ${ }^{13} \mathrm{C}$ NMR spectrum (Figure $2 \mathrm{~b}$ ) shows, in addition to weak signals from residual 2,5$\mathrm{DHB}$, new isotropic resonances in the chemical shift range of aromatic and carbonyl carbons, indicating strong modifications in the hydrogen bond network resulting from the deprotonation of carboxylic groups [33]. Therefore, the shifts observed for the 1:1 mixture may be consistent with an electronic distribution of a carboxylate form obtained by deprotonation of the 2,5-DHB, as described by Fernandez-Bertran et al. [34]. Data obtained in ${ }^{13} \mathrm{C}$ CPMAS experiments performed on 2,5-DHB sodium salt (data not shown) further support this assumption since a similar trend of chemical shift variation was observed for C1, C2, and C5. Acidbase reactions were described to occur upon grinding carboxylic acids with amines [35] or alkali fluorides [34,
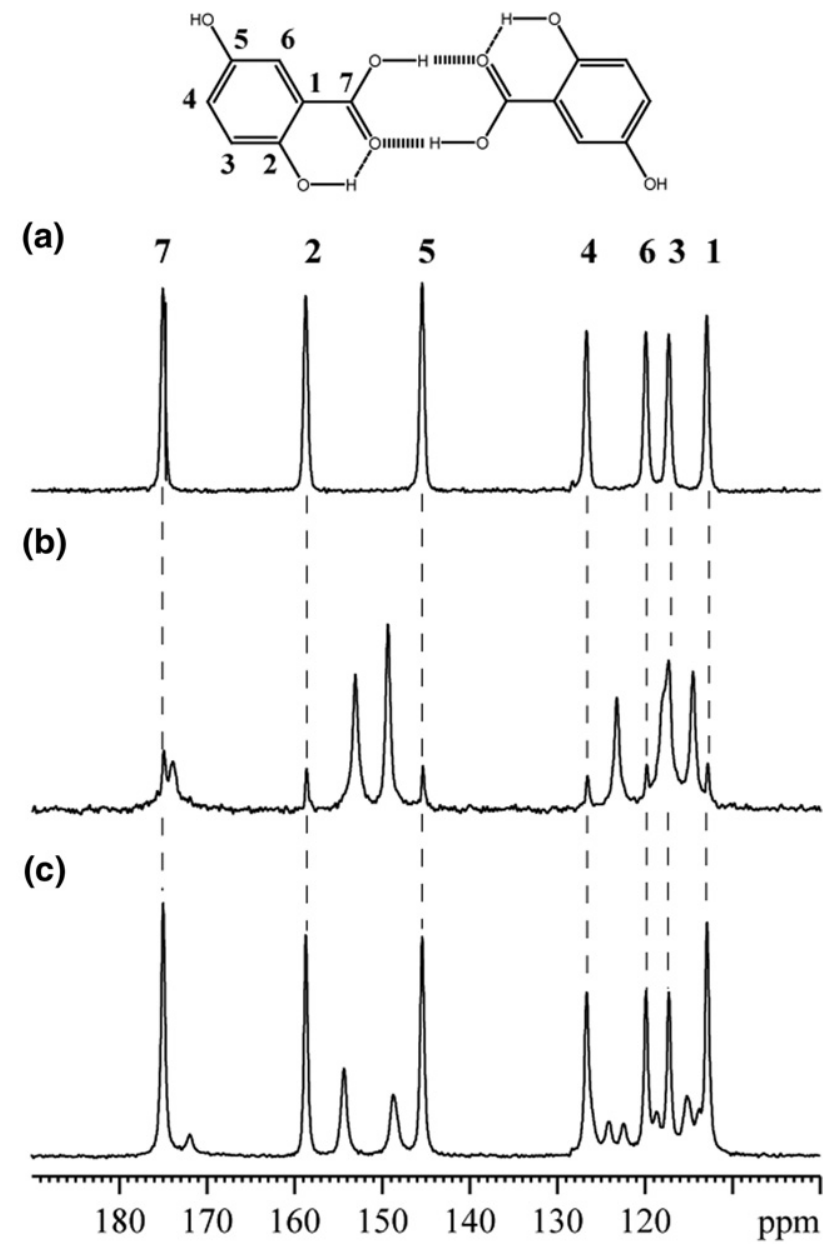

Figure 2. ${ }^{13} \mathrm{C}$ CPMAS spectra of (a) pure 2,5-DHB (inset: solidstate structure of the matrix) and 2,5-DHB/CsF ground mixtures in (b) 1:1 and (c) 5:1 molar ratio. 
36], leading to the formation of hydrogen bonded complexes or to proton transfer reaction with salt formation, respectively. These reactions imply the destruction of intermolecular hydrogen bonds of carboxylic acid dimers. In the case of alkali fluorides [34, 36], milling an acid-alkali fluoride mixture in 1:2 stoichiometric ratio was shown to result in a proton transfer with the formation of a carboxylate and a bifluoride acidic ion $\mathrm{HF}_{2}^{-}$. In such reaction, the driving force was reported to be the formation of the $\mathrm{HF}_{2}^{-}$anion $[34,36]$. Therefore, grinding a mixture of 2,5-DHB and caesium fluoride in a 1:1 ratio would give rise to a solid in which molecular organization (further referred to as Form I) can be described as 2,5-DHB caesium salt while fluoride anions are in strong interaction with $\mathrm{H}_{3} \mathrm{O}^{+}$. It should be noted that for a mixture prepared with dehydrated species, no spectral modification was observed compared with individual starting material, indicating no mechanoinduced structural reorganization during grinding.

For the 5:1 matrix/salt molar ratio, a different molecular organization (further called Form II) is revealed by NMR data. The main ${ }^{19} \mathrm{~F}$ resonance observed at -110 ppm (Figure 1c) actually consists of two overlapping signals, one of which is particularly broad and reflects a large distribution of the chemical shift, suggesting a substantial participation of atmospheric water molecules in the reaction [37]. The ${ }^{133} \mathrm{Cs}$ MAS NMR spectrum still exhibits a typical signal of $\mathrm{Cs}^{+}$coordinated with multiple oxygen atoms [31], but the chemical shift observed for this Form II is weakly down-fielded compared with Form I ( $-5 \mathrm{ppm}$ versus $-11 \mathrm{ppm}$, respectively). This difference is due to a slightly modified organization and participation of ligands in the caesium coordination sphere, in terms of number and distance. This result is further supported by the distinct line shapes, observed for each form in static ${ }^{133} \mathrm{Cs}$ experiments (see Figure S1 in Supplementary Information). Indeed, the chemical shift anisotropy parameters extracted from the line shape analysis highlight the structural difference of both coordination spheres. Clearly,
$\mathrm{Cs}^{+}$coordination environments are different in Form I and Form II. Deconvolution of the ${ }^{13} \mathrm{C}$ CPMAS spectrum revealed up to 12 different resonances. This splitting of some signals is clearly due to two distinct molecules in the asymmetric unit. Comparison of these variations with those observed for Form I indicates that one of the two molecules corresponds to an intermediate molecular structure with a partial proton transfer, while the second one could be assigned to an unmodified 2,5-DHB species. In contrast, solid-state NMR data obtained after grinding the matrix and salt mixture in a 5:1 ratio under nitrogen atmosphere did not show any spectral modification compared with individual starting material.

Further increase of the matrix/salt ratio did not yield new molecular organization, but rather gave rise to a mixture of the Forms I and II, showing a strong sample heterogeneity with locally very low matrix/salt concentration ratios. Moreover, addition of a PEO analyte in the matrix/salt solid mixtures did not induce new NMR signals. Indeed, the same NMR spectra as those presented above were obtained (data not shown), at least when using a matrix/polymer molar ratio of about 250:1, which is appropriate to perform MALDI.

\section{MALDI-TOF-MS Analysis}

Although the matrix/salt ratios were set to low values for the purpose of NMR study and were unusual for MALDI analysis, good quality mass spectra could be obtained. Figure 3 shows PEO 1790 mass spectra obtained for samples prepared in atmospheric conditions using various matrix/salt ratios. Two main differences allow us to distinguish data obtained using the 1:1 matrix/salt ratio (Figure 3a) from those observed for the 5:1 ratio (Figure $3 b$ ). First, when using the lowest matrix/salt ratio, a higher laser fluence $(69 \%$ versus $53 \%$ ) was required to obtain similar signal level by performing the same number of laser shots. In other words, polymer cationization is shown to be more

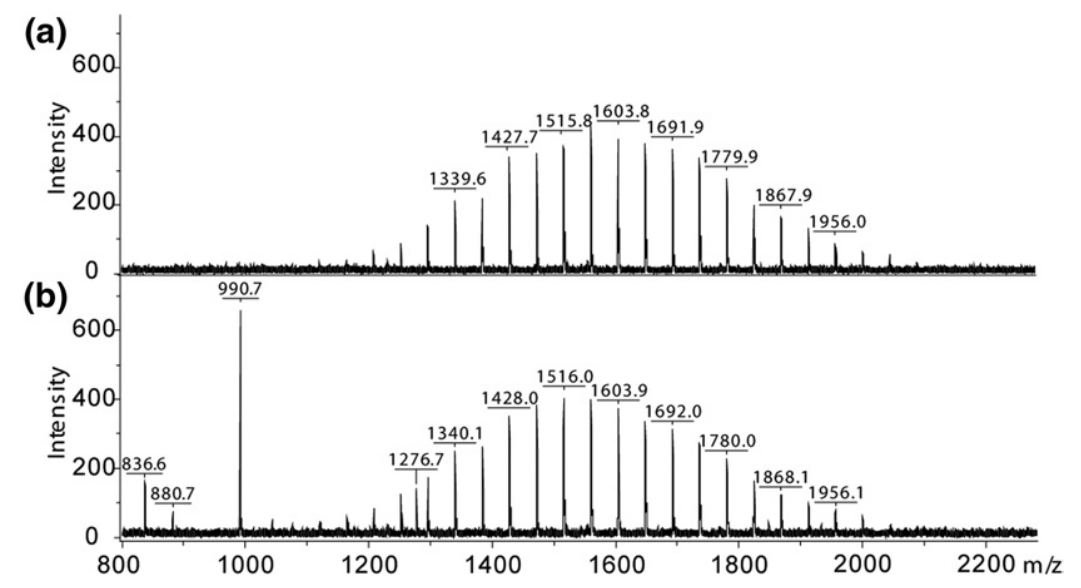

Figure 3. Solvent-free MALDI MS spectra of PEO 1790 using a 2,5-DHB/CsF molar ratio of (a) 1:1, using a $69 \%$ laser fluence and (b) 5:1, using a 53\% laser fluence. The matrix/polymer molar ratio was 250:1 in both cases. 
difficult from Form I, where the matrix is mostly present as a caesium salt, than from Form II, where free molecules of 2,5-DHB were shown to be present together with intermediate molecular structure with a partial proton transfer. This result is consistent with previously reported observation indicating that carboxylic acid matrices perform better in cationizing analytes when converted to a salt at a low level, while $100 \%$ salt conversion leads to very poor MALDI performance [24, $38,39]$. As suggested in these studies, the ability of the matrix to assist analyte desorption may be lost as the matrix becomes a salt. The other difference observed between data in Figure $3 a$ and $b$ is the presence of additional peaks in the low $m / z$ range of the mass spectra obtained from the 5:1 mixture. Based on $\mathrm{m} / \mathrm{z}$ values, the intense signal at $m / z 991$ could be assigned to a cationized cluster of the matrix caesium salt, namely $[2,5-\mathrm{DHB}-\mathrm{H}+\mathrm{Cs}]_{3} \mathrm{Cs}^{+}$. The homologue cationized cluster consisting of four salt units gives rise to the signal detected at $m / z$ 1277. Similarly, the weak abundance peaks at $m / z 837$ and 881 would correspond to mixed cationic clusters, $\left[2,5-\mathrm{DHB}-\mathrm{H}+\mathrm{Na}_{4} \mathrm{Cs}^{+}\right.$and $[2,5-\mathrm{DHB}-\mathrm{H}+\mathrm{Cs}]_{2}[2,5-\mathrm{DHB}-\mathrm{H}+\mathrm{Na}] \mathrm{Cs}^{+}$, respectively. This last result indicates the presence of residual sodium in the matrix sample. Interestingly, although the matrix was shown to predominantly exist as its caesium salt form in the 1:1 mixture, salt agglomeration was not observed to occur from this Form I. This could either suggest low intermolecular interaction in Form I or the availability of a limited number of $\mathrm{Cs}^{+}$ cations, which would preferentially attach to polymer molecules.

Since significant differences were observed in solidstate NMR while changing the preparation conditions of the MALDI samples (e.g., atmospheric conditions versus nitrogen atmosphere), MALDI MS experiments were also performed for samples prepared in dried conditions. However, it should be noted that although prepared under strictly anhydrous conditions, samples might experience some contact with the atmosphere during the transfer of the MALDI target from the glove box to the MALDI source. MALDI efficiency was systematically found to be higher for samples prepared in the absence of water molecules. Using a 10:1 matrix/ salt mixture in which both Form I and Form II are present, PEO 1790 signal could be obtained from the dried MALDI sample with a laser fluence of $43 \%$ (Figure 4a), while a 52\% laser fluence was required to obtain peaks of similar intensity from the sample prepared at atmospheric conditions (data not shown). Solid-state NMR data have revealed that atmospheric water molecules actually play a role in the molecular organization of the solvent-free MALDI samples. Deprotonation of 2,5-DHB, availability of caesium cations, and their interaction with the matrix, together with solvation of the salt counter-anion, were indeed mainly induced by grinding all raw materials in atmospheric conditions, showing the crucial role of water in this reorganization of the solid. As a result, preparing samples under dried conditions is shown in our case to benefit to the MALDI process for polymer cationization. Results obtained from samples prepared under dried conditions also show that such a molecular organization is detrimental to the MALDI process for polymer cationization. Interestingly, leaving the "dried" sample on the MALDI target in the atmosphere of the laboratory for $24 \mathrm{~h}$ was sufficient to induce some molecular reorganization, since a higher laser fluence (47\%) was required to obtain the targeted PEO 1790 signal (Figure $4 b)$. It should also be noted again that cationized salt clusters, which were poorly observed in the low $\mathrm{m} / \mathrm{z}$ range from the "dried" sample, are readily formed after

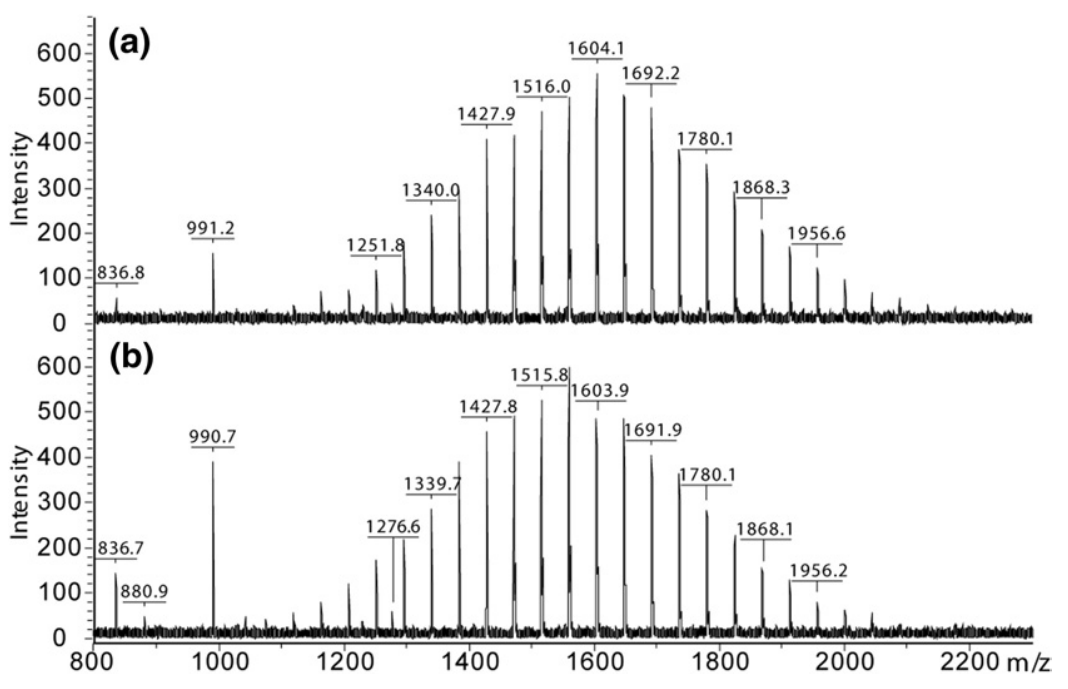

Figure 4. Solvent-free MALDI MS spectra of PEO 1790 using a 2,5-DHB/CsF molar ratio of 10:1 prepared (a) in dried conditions (laser fluence: 43\%) and (b) after leaving the dried sample in atmospheric conditions for $24 \mathrm{~h}$ (laser fluence: 47\%). The matrix/polymer molar ratio was 250:1 in both cases. 
the MALDI sample has been exposed to atmospheric water.

\section{Acknowledgments}

The authors acknowledge support for this work by the French Research Agency (ANR-06-JCJC-0112). L.C. acknowledges support from Spectropole, the Analytical Facility of Aix-Marseille University, by allowing a special access to the instruments purchased with European funding (FEDER OBJ2142-3341).

\section{Appendix A Supplementary Material}

Supplementary material associated with this article may be found in the online version at doi:10.1016/j.jasms. 2009.06.021.

\section{References}

1. Montaudo, G.; Montaudo, M. S.; Samperi, F. Matrix-Assisted Laser Desorption/Ionization Mass Spectrometry of Polymers (MALDI-MS). In Mass Spectrometry of Polymers; CRC Press: New York, 2002; pp 419-522.

2. Pasch, H.; Schrepp, W. Mass Spectrometry of Synthetic Polymers; Springer: New York, 2003.

3. Wallace, W. E.; Bauer, B. J.; Blair, W. R.; Flynn, K. M.; Gutman, C. M.; Kearsley, A. J. http:/ / polymers.msel.nist.gov/maldirecipes/index.cfm.

4. Skelton, R.; Dubois, F.; Zenobi, R. A MALDI sample preparation method suitable for insoluble polymers. Anal. Chem. 2000, 72, 17071710 .

5. Trimpin, S.; Rouhanipour, A.; Az, R.; Rader, H. J.; Mullen, K. New Aspects in Matrix-Assisted Laser Desorption/Ionization Time-of-Flight Mass Spectrometry: A Universal Solvent-Free Sample Preparation. Rapid Commun. Mass Spectrom. 2001, 15, 1364-1373.

6. Doktycz, S. J.; Savickas, P. J.; Krueger, D. A. Matrix Sample Interactions in Ultraviolet Laser-Desorption of Proteins. Rapid Commun. Mass Spectrom. 1991, 5, 145-148.

7. Preston, L. M.; Murray, K. K.; Russell, D. H. Reproducibility and Quantitation of Matrix-Assisted Laser-Desorption Ionization MassSpectrometry-Effects of Nitrocellulose on Peptide Ion Yields. Biol. Mass Spectrom. 1993, 22, 544-550.

8. Chan, T. W. D.; Colburn, A. W.; Derrick, P. J.; Gardiner, D. J.; Bowden, M. Suppression of Matrix Ions in Ultraviolet-Laser Desorption-Scanning Electron-Microscopy and Raman-Spectroscopy of the Solid Samples. Org. Mass Spectrom. 1992, 27, 188-194.

9. Westman, A.; Huthfehre, T.; Demirev, P.; Sundqvist, B. U. R. Sample Morphology Effects in Matrix-Assisted Laser-Desorption Ionization Mass-Spectrometry of Proteins. J. Mass Spectrom. 1995, 30, 206-211.

10. Gusev, A. I.; Wilkinson, W. R.; Proctor, A.; Hercules, D. M. Improvement of Signal Reproducibility and Matrix/Co-Matrix Effects in MALDI Analysis. Anal. Chem. 1995, 67, 1034-1041.

11. Horneffer, V.; Reichelt, R.; Strupat, K. Protein Incorporation into MALDI-Matrix Crystals Investigated by High Resolution Field Emission Scanning Electron Microscopy. Int. J. Mass Spectrom. 2003, 226, 117-131.

12. Hanton, S. D.; Hyder, I. Z.; Stets, J. R.; Owens, K. G.; Blair, W. R.; Guttman, C. M.; Giuseppetti, A. A. Investigations of Electrospray Sample Deposition for Polymer MALDI Mass Spectrometry. J. Am. Soc. Mass Spectrom. 2004, 15, 168-179.

13. Horneffer, V.; Forsmann, A.; Strupat, K.; Hillenkamp, F.; Kubitscheck, U. Localization of Analyte Molecules in MALDI Preparations by Confocal Laser Scanning Microscopy. Anal. Chem. 2001, 73, 1016-1022.

14. Horneffer, V.; Strupat, K.; Hillenkamp, F. Localization of Noncovalent Complexes in MALDI Preparations by CLSM. J. Am. Soc. Mass Spectrom. 2006, 17, 1599-1604

15. Hanton, S. D.; Clark, P. A. C.; Owens, K. G. Investigations of MatrixAssisted Laser Desorption/Ionization Sample Preparation by Time-of-
Flight Secondary Ion Mass Spectrometry. J. Am. Soc. Mass Spectrom. 1999, 10, 104-111.

16. Garden, R. W.; Sweedler, J. V. Heterogeneity Within MALDI Samples as Revealed by Mass Spectrometric Imaging. Anal. Chem. 2000, 72, 30-36.

17. Mele, A.; Malpezzi, L. Noncovalent Association Phenomena of 2,5Dihydroxybenzoic Acid with Cyclic and Linear Oligosaccharides. A Matrix-Assisted Laser Desorption/Ionization Time-of-Flight Mass Spectrometric and X-ray Crystallographic Study. J. Am. Soc. Mass Spectrom. 2000, $11,228-236$

18. Strupat, K.; Kampmeier, J.; Horneffer, V. Investigations of 2,5-DHB and Succinic Acid as Matrices for UV and IR MALDI. Part II: Crystallographic and Mass Spectrometric Analysis. Int. J. Mass Spectrom. 1997, 169, 43-50.

19. Hanton, S. D.; McEvoy, T. M.; Stets, J. R. Imaging the Morphology of Solvent-Free Prepared MALDI Samples. J. Am. Soc. Mass Spectrom. 2008, $19,874-881$

20. Andrew, E. R. Magic Angle Spinning. In Encyclopedia of NMR; Grant, D. M.; Harris, R. K., Eds., Wiley: Chichester, 1996; pp 2891-2901.

21. Schaefer, J.; Stejskal, E. O. C. -13 Nuclear Magnetic-Resonance of Polymers Spinning at Magic Angle. J. Am. Chem. Soc. 1976, 98, 10311032.

22. Sroka-Bartnicka, A.; Olejniczak, S.; Sochacki, M.; Biela, T.; Potrzebowski, M. J. Solid-State NMR Spectroscopy as a Tool Supporting Optimization of MALDI-TOF MS Analysis of Polylactides. J. Am. Soc. Mass Spectrom. 2009, 20, 67-72.

23. Spickermann, J.; Martin, K.; Rader, H. J.; Mullen, K.; Schlaad, H.; Muller A. H. E.; Kruger, R. P. Quantitative Analysis of Broad Molecular Weight Distributions Obtained by Matrix-Assisted Laser Desorption Ionization Time-of-Flight Mass Spectrometry. Eur. Mass Spectrom. 1996, 2, 161-165.

24. Knochenmuss, R.; Lehmann, E.; Zenobi, R. Polymer Cationization in Matrix-Assisted Laser Desorption/Ionization. Eur. Mass Spectrom. 1998, 4, 421-426.

25. Massiot, D.; Fayon, F.; Capron, M.; King, I.; Le Calve, S.; Alonso, B.; Durand, J. O.; Bujoli, B.; Gan, Z. H.; Hoatson, G. Modeling One- and Two-Dimensional Solid-State NMR Spectra. Magn. Reson. Chem. 2002, 40, 70-76.

26. Gross, U · Rudiger, S.; Grimmer, A. R · Kemnitz, E. F. -19-NMR Solid State Investigations of Monovalent Alkali Metal Fluorides and TetraAlkylammonium Fluorides. J. Fluorine Chem. 2002, 115, 193-199.

27. Hayashi, S.; Hayamizu, K. Accurate Determination of NMR ChemicalShifts in Alkali-Halides and Their Correlation with Structural Factors. Bull. Chem. Soc. Jpn. 1990, 63, 913-919.

28. Miller, J. M. Fluorine-19 Magic-Angle Spinning NMR. Progr. Nucl. Magn. Reson. Spectrosc. 1996, 28, 255-281.

29. Haisa, M.; Kashino, S.; Hanada, S. I.; Tanaka, K.; Okazaki, S.; Shibagaki, M. The Structures of 2-Hydroxy-5-Methylbenzoic Acid and Dimorphs of 2,5-Dihydroxybenzoic Acid. Acta Crystallogr. Sect. B Struct. Sci. 1982, $38,1480-1485$.

30. Asseid, F. M.; Miller, J. M.; Clark, J. H. FT-IR and Si-29, Al-27, and F-19 Mas NMR-Studies of the Adsorption of CdF2, ZnF2, and CuF2 onto Montmorillonite K10-Activity Towards Friedel-Crafts Alkylation. Can. J. Chem. 1992, 70, 2398-2404.

31. Wong, A.; Sham, S.; Wang, S. N.; Wu, G. A Solid-State Cs-133 Nuclear Magnetic Resonance and X-ray Crystallographic Study of Cesium Complexes with Macrocyclic Ligands. Can. J. Chem. 2000, 78, 975-985.

32. Wiesbrock, F.; Schmidbaur, H. Crystal Structures of Rubidium and Cesium Anthranilates and Salicylates. Inorg. Chem. 2003, 42, 7283-7289.

33. Claramunt, R. M.; Garcia, M. A.; Lopez, C.; Elguero, J. The Structure of 3,5-Dimethylpyrazole/Carboxylic Acids Co-Crystals. ARKIVOC 2005, 91-101.

34. Fernandez-Bertran, J.; Reguera, E. Proton Transfer in the Solid State: Mechano-Chemical Reactions of Fluorides with Acidic Substances. Solid State Ionics 1998, 112, 351-354.

35. Fernandez-Bertran, J.; Alvarez, J. C.; Reguera, E. Proton Transfer in the Solid State: Reactions of Organic Acids and Amines. Solid State Ionics 1998, 106, 129-135.

36. Paneque, A.; Reguera, E.; Fernandez-Bertran, J.; Yee-Madeira, H. Mechano-Chemical Reactions of Fluorides with Hemin. J. Fluorine Chem. 2002, 113, 1-5.

37. Gerken, M.; Boatz, J. A.; Kornath, A.; Haiges, R.; Schneider, S.; Schroer, T.; Christe, K. O. The F-19 NMR shifts are not a measure for the nakedness of the fluoride anion. I. Fluorine Chem. 2002, 116, 49-58.

38. Dogruel, D.; Nelson, R. W.; Williams, P. The effects of matrix $\mathrm{pH}$ and cation availability on the matrix-assisted laser desorption ionization mass spectrometry of poly(methyl methacrylate). Rapid Commun. Mass Spectrom. 1996, 10, 801-804.

39. King, R. C.; Owens, K. G. Proceedings of the 43rd Annual Conference on Mass Spectrometry and Allied Topics; Atlanta, GA, 1995. 